\title{
Ulipristal Acetate: Medical Management for Benign Metastasizing Leiomyoma?
}

\author{
Adina Schwartz, MD*, Olivia Carpinello, MD and Alan DeCherney, MD \\ Program in Reproductive Endocrinology and Gynecology, Eunice Kennedy Shriver NICHD, USA
}

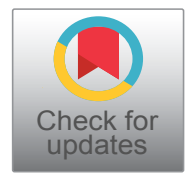

*Corresponding author: Adina Schwartz, MD, Program in Reproductive Endocrinology and Gynecology, Eunice Kennedy Shriver NICHD, 10 Center Drive Rm 5-5570, Bethesda, MD, 20892, USA, Tel: (732)-266-7590, Fax: (301)-480-0665

\begin{abstract}
Objective: To describe the use of ulipristal acetate in an attempt to medically treat benign metastasizing leiomyoma (BML)

Design: Case report.

Background: Benign metastasizing leiomyoma (BML) is a rare disease characterized by smooth muscle tumors found outside of the uterus. Treatment options include surgery, embolization, and medical management. This case describes the use of ulipristal acetate in an attempt to medically manage an inoperable case of BML.

Patient: A 40-year-old female presented with abdominal pain, urinary frequency, and decreased appetite several years after a total hysterectomy and was ultimately diagnosed with BML. She was initially treated with leuprolide acetate, letrozole and embolization as she was not a surgical candidate.

Intervention: Ulipristal acetate was added to her existing regimen.

Results: The patient's mass continued to enlarge and she developed transaminitis. Ulipristal acetate was discontinued due to the elevated LFTs and lack of improvement in tumor size.

Conclusion: Ulipristal acetate did not prove to be an effective form of treatment for BML in this patient. Additional cases of BML treated with ulipristal acetate need to be studied to help determine the true efficacy, safety, and optimal regimen. It is unknown whether a lower dose or longer course of ulipristal acetate would be an effective treatment option for BML if surgery is not an option.
\end{abstract}

\section{Keywords}

Benign metastasizing leiomyoma, Ulipristal acetate, Drug induced liver injury

\begin{abstract}
Abbreviations
BML:Benign metastasizing leiomyoma; $\mathrm{GnRH}$ :Gonadotropin releasing hormone; SERM: Selective estrogen receptor modulator; CT: Computerized tomography; IVC: Inferior vena cava; IM: Intramuscular; IR: Interventional radiology; DILI: Drug induced liver injury; PRAC: Pharmacovigilance risk assessment committee; LFT: Liver function test; AST: Aspartate aminotransferase; ALT: Alanine aminotransferase
\end{abstract}

\section{Introduction}

Benign metastasizing leiomyoma (BML) is a rare disease characterized by smooth muscle tumors found outside of the uterus. Tumors are most commonly found in the lungs, but can also be seen in the abdomen, lymph nodes, soft tissue, heart, spine and bone [1,2]. BML was first described by Steiner in 1939 [3], and there have since been approximately 200 cases documented. Most women with BML are premenopausal and have a history of prior uterine surgery (dilation and curettage, myomectomy, or hysterectomy) $[4,5]$. Some present with respiratory symptoms, but women are often asymptomatic, and the diagnosis is usually made with imaging and pathology $[1,6]$.

BML has some cancer-like properties with remote metastases but is slow growing with low mitotic counts and does not show nuclear pleomorphism. This differentiates it from its more aggressive counterpart, leiomyosarcoma $[7,8]$. Furthermore, BML has been shown to be molecularly similar to benign leiomyomas. They have been shown to share the same clonality and both express estrogen and progesterone receptors, similar to uterine smooth muscle $[7,9-11]$. This all supports the

Citation: Schwartz A, Carpinello O, DeCherney A (2020) Ulipristal Acetate: Medical Management for Benign Metastasizing Leiomyoma?. Obstet Gynecol Cases Rev 7:184. doi.org/10.23937/23779004/1410184

Accepted: December 01, 2020: Published: December 03, 2020

Copyright: (C) 2020 Schwartz A, et al. This is an open-access article distributed under the terms of the Creative Commons Attribution License, which permits unrestricted use, distribution, and reproduction in any medium, provided the original author and source are credited. 
notion that $\mathrm{BML}$ is related to benign uterine leiomyomas. There are several theories to explain the pathophysiology of BML including de novo proliferation, seeding after mechanical instrumentation, vascular or lymphatic spread, deposits from intravenous leiomyoma, metastasis of uterine leiomyoma, and hormonally induced metaplastic transformation $[1,12,13]$.

Several different approaches have been used to treat BML including surgery, embolization, and medical management. Debulking procedures attempt to remove metastatic lesions whereas an oophorectomy acts as surgical castration. Embolization is considered a good option for a patient that has a single lesion but is not effective for a patient with multiple lesions. Categories of hormonal therapy used to treat BML include GnRH agonists, aromatase inhibitors and selective estrogen receptor modulators (SERMs). GnRH agonists, such as leuprolide acetate, act by decreasing endogenous gonadotropin secretion, and therefore decrease estrogen and progesterone levels. Aromatase inhibitors, such as letrozole, block the final step in the conversion of androgen to estrogen, thereby decreasing estrogen production. Selective estrogen receptor modulators, such as raloxifene, function as estrogen receptor agonists in skeletal, cardiovascular and central nervous systems but as estrogen receptor antagonists in the breast and uterus [12].

A newer category of hormone therapy is the selective progesterone receptor modulator, now known as ulipristal acetate. Ulipristal acetate has been used as a form of emergency contraception for almost ten years. It has more recently been proven to be an effective treatment for leiomyomata by successfully decreasing the volume of fibroids, the amount of bleeding, and pain secondary to fibroids [14]. It functions by remodeling the extracellular matrix and inducing apoptosis. Thus, due to the similarities between BML and fibroids, this medication may, theoretically, provide benefit in the medical management of BML. There have been three case reports of ulipristal acetate being used to treat BML. In one instance, after using $5 \mathrm{mg}$ ulipristal acetate daily for 16 months, the patient symptomatically improved but the mass continued to increase in size [15]. In the second case, the patient improved symptomatically and her nodules regressed over time after 5 years of ulipristal [16]. In the third case, the study did not look at the success of treatment but examined the long-term endometrial effects of taking ulipristal acetate. In this case, no malignant or premalignant endometrial pathology was identified in biopsies over a five year course of treatment [17]. Though the data is limited, this medication may be useful in patients who are not surgical candidates and have not responded to other forms of medical management. This case describes our experience with the use of ulipristal acetate in the medical management of inoperable BML.

\section{Case Report}

A 40-year-old G1P1 initially presented with worsening abdominal pain, urinary frequency, and decreased appetite that developed approximately three years after a total hysterectomy was performed for uterine fibroids and menorrhagia. Initial CT scan showed a large mass in the left side of the abdomen and pelvis measuring $19.4 \times$ $16.1 \mathrm{~cm}$. The mass was also noted to involve the iliac arteries and displaced the left kidney, pancreas, stomach, and bladder. Multiple pulmonary nodules were seen in the right upper and lower lobes, the largest of which measured $4 \mathrm{~cm}$. A CT-guided biopsy was performed on the abdomino-pelvic mass and histopathology was estrogen receptor positive, consistent with benign leiomyoma. This, in combination with pulmonary nodules, led to a diagnosis of benign metastasizing leiomyoma. CT scan also identified a thrombus (it was unknown whether this was clot versus tumor) in the inferior vena cava (IVC). An IVC filter was placed and the patient was started on therapeutic enoxaparin $100 \mathrm{mg}$ daily.

As the pelvic mass was also noted to involve the iliac vessels, it was felt that surgical debulking could not safely be performed. Medical management was started with leuprolide acetate $22.5 \mathrm{mg} I \mathrm{IM}$ every 3 months and letrozole $2.5 \mathrm{mg}$ daily. Additionally, she was treated with four embolizations of the pelvic mass by interventional radiology (IR) over the course of the next six months. Her symptoms continued to worsen, and the patient required morphine sulfate and oxycodone daily for pain control. Serial CT scans revealed both the pelvic mass and pulmonary nodules increased in size despite eight months of medical treatment, and the patient was referred to our care for further management.

On initial exam, the patient's abdomen was soft, nontender, and a mass was appreciated in the left abdomen and pelvis approximately the size of a 20-week uterus. Ulipristal acetate $30 \mathrm{mg}$ daily was added to the existing regimen of leuprolide acetate and letrozole.

\section{Materials and Methods}

A single subject was followed at a medical research center. The main outcome measure was the size of the mass monitored with CT scans. The secondary outcome measure was liver function determined by measuring liver enzymes (AST and ALT). The mass size and liver enzymes 6 weeks after starting treatment with ulipristal acetate were compared to the values prior to treatment.

\section{Results}

At her six-week follow up visit, the patient reported feeling dull left-sided abdominal pain that was well controlled with narcotics, but overall improved compared to before starting the ulipristal acetate. On exam, the mass was palpated to be the equivalent of a 28 -week uterus. A repeat CT scan 10 weeks after the prior scan 
showed the mass had enlarged to $31.4 \times 15.6 \mathrm{~cm}$, surrounded the left renal artery, abutted the left renal vein, and surrounded at least half of the aorta and left iliac branches. There was also a separate mass in the right abdomen measuring $6.9 \times 5.0 \mathrm{~cm}$. Serum laboratory values revealed transaminitis. Her AST and ALT had risen from 20 and $30 \mathrm{U} / \mathrm{L}$ pre-ulipristal acetate to 41 and 85 $U / L$, respectively. The decision was made to continue leuprolide acetate and letrozole but to discontinue the ulipristal acetate given the transaminitis and increase in tumor size.

\section{Discussion}

This case report presents a patient who was given ulipristal acetate in an attempt to treat her refractory case of benign metastasizing leiomyoma. This is only the fourth report describing treatment of this rare condition with ulipristal acetate. Currently, the only dose of ulipristal acetate available on the market in the United States is $30 \mathrm{mg}$, which is the dose recommended for emergency contraception. However, the recommended dose for leiomyomata is 5-10 mg daily. Our patient was started on $30 \mathrm{mg}$ daily, as that was the dose available at the pharmacy. At her six week follow up visit, the mass had increased in size. Thus, it is unknown whether the ulipristal acetate was ineffective due to the use of a different dosing regimen, a shorter duration of treatment than was used in other case reports, or whether the medication itself was simply ineffective in our patient.

An additional complication in this case was the development of mild transaminitis over the six weeks of treatment with ulipristal acetate. Ulipristal acetate $5 \mathrm{mg}$ tablets have been available in Europe for the treatment of leiomyomata since 2012 (Esmya). However, eight cases of drug-induced liver injury (DILI) were potentially linked to Esmya, including four cases requiring liver transplantation. The pharmacovigilance risk assessment committee (PRAC) performed a review of all available data from clinical trials and post-marketing and, while they could not find evidence of hepatic toxicity linked to the drug, concluded that it may have contributed to a few rare cases of DILI. Several recommendations were then made including only using Esmya for patients who are not surgical candidates, avoiding use for patients with known liver disorders, and performing liver function tests before, during, and after treatment [18]. LFTs were performed in our patient due to this potential complication and a rise in AST/ALT led to the discontinuation of the medication after six weeks.

BML is known to be molecularly similar to benign leiomyomata. Therefore, ulipristal acetate has been proposed as a hormonal treatment for BML. It has led to improved symptoms in other case reports and has reduced the size of masses in a single case report [15-17]. Unfortunately, due to the development of transaminitis in our patient, she was unable to continue ulipristal ac- etate treatment. Furthermore, a progression in tumor size, rather than reduction, was seen.

For this rare condition with few treatment options, it remains unknown whether ulipristal acetate can provide benefit when surgery is not an option or other medical therapies have failed. However, if ulipristal acetate is used, a lower dose should be considered, and close liver function monitoring is necessary as the efficacy, safety, and optimal regimen have not yet been determined. Additional cases of BML treated with ulipristal acetate need to be studied and described in order to ascertain whether the theoretical benefit translates into actual benefit in this rare condition.

\section{Financial Support}

The authors received no financial support for the research, authorship, or publication of this article.

\section{Authors' Contribution}

All authors contributed equally to this article.

\section{References}

1. Awonuga AO, Shavell VI, Imudia AN, Rotas M, Diamond MP, et al. (2010) Pathogenesis of benign metastasizing leiomyoma. Obstet Gynecol Surv 65: 189-195.

2. Yuan X, Sun $Y$, Jin $Y, X u$ L, Dai H, et al. (2019) Multiple organ benign metastasizing leiomyoma: $A$ case report and literature review. Obstet Gynaecol Res 45: 2132-2136.

3. Steiner PE (1939) Metastasizing fibroleiomyoma of the uterus: Report of a case and review of literature. Am J Path 15: $89-110$

4. Pacheco-Rodriguez G, Taveira-DaSilva AM, Moss J (2016) Benign metastasizing leiomyoma. Clin Chest Med 37: 589595.

5. Barnas E, Ksiazek M, Ras R, Skret A, Skret-Magierlo J, et al. (2017) Benign metastasizing leiomyoma: A review of current literature in respect to the time and type of previous gynecological surgery. PLoS One 12: e0175875.

6. Asumu H, Estrin Y, Mohammed TL, Verma N (2017) Benign metastasizing leiomyoma. Curr Probl Diagn Radiol 46: 257-259.

7. Patton KT, Cheng L, Papavero V, Blum MG, Yeldandi AV, et al. (2006) Benign metastasizing leiomyoma: Clonality, telomere length and clinicopathologic analysis. Mod Pathol 19: $130-140$

8. Ip PP, Tse KY, Tam KF (2010) Uterine smooth muscle tumors other than the ordinary leiomyomas and leiomyosarcomas: A review of selected variants with emphasis on recent advances and unusual morphology that may cause concern for malignancy. Adv Anat Pathol 17: 91-112.

9. Jautzke G, Muller-Ruchholtz E, Thalmann U (1996) Immunohistological detection of estrogen and progesterone receptors in multiple and well differentiated leiomyomatous lung tumors in women with uterine leiomyomas (so-called benign metastasizing leiomyomas). A report on 5 cases. Pathol Res Pract 192: 215-223.

10. Esteban JM, Allen WM, Schaerf RH (1999) Benign metastasizing leiomyoma of the uterus: Histologic and immunohistochemical characterization of primary and metastatic lesions. Arch Pathol Lab Med 123: 960-962. 
11. Tietze L, Gunther K, Horbe A, C Pawlik, B Klosterhalfen, et al. (2000) Benign metastasizing leiomyoma: A cytogenetically balanced but clonal disease. Hum Pathol 31: 126-128.

12. Rivera JA, Christopoulos S, Small D, Trifiro M (2004) Hormonal manipulation of benign metastasizing leiomyomas: Report of two cases and review of the literature. Clin Endocrinol Metab 89: 3183-3188.

13. Robboy SJ1, Bentley RC, Butnor K, Anderson MC (2000) Pathology and pathophysiology of uterine smooth-muscle tumors. Environ Health Perspect 5: 779-784.

14. Donnez J, Vazquez F, Tomaszewski J, Nouri K, Bouchard $P$, et al. (2014) Long-term treatment of uterine fibroids with ulipristal acetate. Fertil Steril 101: 1565-1573.

15. Kortekaas KE, Pelikan HMP (2018) Non-surgical interven- tion for retroperitoneal lymphogenic and pulmonary metastases of a benign leiomyoma: Treatment with ulipristal acetate. BMJ Case Rep.

16. Lewis El, Chason RJ, DeCherney AH, Armstrong A, Elkas $\mathrm{J}$, et al. (2013) Novel hormone treatment of benign metastasizing leiomyoma: An analysis of five cases and literature review. Fertil Steril 99: 2017-2024.

17. Levy G, Elkas J, Armstrong AY, Nieman LK (2016) Endometrial effects of prolonged therapy with the selective progesterone receptor modulator ulipristal acetate: A case report. J Reprod Med 61: 159-162.

18. Donnez J, Arriagada P, Marciniak M, Larrey D (2018) Liver safety parameters of ulipristal acetate for the treatment of uterine fibroids: A comprehensive review of the clinical development program. Expert Opin Drug Saf 17: 1225-1232. 